\title{
CONVERGENCE OF POSITIVE FUNCTIONS IN THE SPACE ON MIKUSIŃSKI OPERATORS
}

\author{
T. K. BOEHME
}

1. Introduction. We shall show some properties of convergent sequences of positive functions in the space of Mikusinski operators. In particular we shall show that a sequence of non-negative integrable functions can converge to a function which is strictly negative. We shall consider the positive cone $\beta=\{p \mid p \in \mathcal{L}, p(t) \geqq 0$ a.e. $t \geqq 0\}$ in the space $\mathscr{L}$ of locally integrable functions on the half-line, and we are interested in the image of this cone under convolution; that is, the set $\phi \mathcal{P}$ which consists of all the convolution products $\phi p$, where

$$
(\phi p)(t)=\int_{0}^{t} \phi(t-u) p(u) d u, \quad t \geqq 0, p \in \mathcal{P} .
$$

We shall give several conditions on $\phi$ which are necessary and sufficient to insure that the closure in $\mathscr{L}$ of $\phi \mathcal{P}$ is the set $\phi \mathfrak{N}$ - the set of all convolution products of $\phi$ with non-negative locally finite Baire measures. It is shown that if the closure in $\mathscr{L}$ of $\phi \mathcal{P}$ properly contains $\phi \mathfrak{T}$ then it is all of $\mathscr{L}$ (when $\phi$ vanishes on no neighborhood of the origin).

If $f_{n}$ is convergent in $\mathscr{L}$ to $f$ the sequence $q_{n}$ obtained by convolving each $f_{n}$ with some fixed $\phi$ is convergent to the convolution product of $\phi$ with $f$. In this sense convolution with $\phi$ provides a regular summability method and Lemma 1 is a Tauberian theorem; it shows that for bounded sequences all summability methods (i.e. all convergence factors $\phi$ ) have the same strength. In Lemma 2 the boundedness condition is replaced by positivity; in this case an additional condition must be placed on the function $\phi$ to get the Tauberian theorem. Namely, there must exist a nonzero function $k$ such that the convolution of $\phi$ with $k$ is non-negative. In the theorem this condition is shown to be necessary as well as sufficient, and several equivalent conditions are given.

2. Notation. By $\&$ we mean the real vector space of real valued functions on the half-line $t \geqq 0$ which are Lebesgue integrable on each interval $[0, T], T>0$. As usual we identify functions which are equal except for a set of measure zero (a.e.). Addition in $\&$ is the usual pointwise addition, and the field of scalars is the field of real numbers. The space $L[0, T]$ is, as usual, the vector space of functions which are

Received by the editors July 14, 1964. 
Lebesgue integrable on $[0, T]$. Elements of $\mathscr{L}$ will frequently be considered to be in $L[0, T]$ by identifying $f$ with its image under the natural mapping of $\mathscr{L}$ into $L[0, T], T>0$; operators on $\mathscr{L}$ to $\mathscr{L}$ will be considered also to be operators on $L[0, T]$ to $L[0, T], T>0 . \mathscr{L}$ is given a topology by means of the collection of semi-norms $\|f\|_{T}$ $=\int_{0}^{T}|f|, T>0$. A set or a sequence is bounded in $\mathscr{L}$ if and only if it is bounded in $L[0, T]$ for every $T>0$.

We shall use the notation of the Mikusiński operational calculus to the extent that

(i) juxtaposition of functions denotes convolution; thus, $r=\phi f$ is the element of $\mathscr{L}$ obtained by the convolution of $\phi$ with $f$;

(ii) $h$ is the function in $\mathscr{L}$ which is one for every $t \geqq 0$, and $s=h^{-1}$ is the Mikusiński differentiation operator. The operator $e^{-\lambda_{s}}, \lambda>0$ is the shift operator; thus $g=f e^{-\lambda s}$ is the function which is equal to zero on $[0, \lambda]$ and $g(t)=f(t-\lambda)$ for $t \geqq \lambda$.

In order to avoid minor special arguments the symbol $\phi$ will invariably denote the element of $\mathscr{L}$ which vanishes on no neighborhood of the origin; that is, $\int_{0}^{t}|\phi|>0$ for $t>0$. If there is a nonzero function $k$ such that $(k \phi)(t) \geqq 0$ for all $t \geqq 0$ it is easy to see (by shifting $k$ to the left if necessary) that there is a $k$ such that $k \phi$ is non-negative and vanishes in no neighborhood of the origin. In fact we can take $k \phi$ to be strictly positive (or even strictly increasing and positive) on $t>0$ by integrating the original $k$ once (or twice). We shall take the statement $\phi$ can be made positive by convolution to mean that there is a $k$ such that $(\phi k)(t)>0$ for $t>0$. We will say $\phi$ has a positive real part under convolution if there is a $k$ such that $\operatorname{Re}(\phi k)(t)>0$ for all $t>0$.

We shall repeatedly use the basic theorem of Titchmarsh [1] on the uniqueness of convolution products in the form which says that if $(\phi f)(t)=0$ a.e. on $[0, T]$ then $f(t)=0$ a.e. on $[0, T]$, and we shall use the theorem which says that $\phi \mathscr{L}$, the set of convolution products of elements in $\mathscr{L}$ with $\phi$, is dense in $\mathscr{L}$ [2], [3], [4].

The symbol $\mu$ shall denote a locally finite, non-negative Baire measure on the half-line. For each $f \in \mathcal{L}$ the convolution product $f \mu$ defined by

$$
(f \mu)(t)=\int_{0}^{t} f(t-u) d \mu(u), \quad t \geqq 0,
$$

is in $\mathscr{L}$. The norm of a function in $L[0, T]$ is equal to its norm when it is considered as an absolutely continuous Baire measure, and bounded sets in this norm are compact in the weak star topology for the Baire measures-the topology of pointwise convergence for the continuous linear functionals on the space of continuous functions on $[0, T]$ with the sup norm. 
3. The main theorem. The functions in this section will all be real valued unless it is specified otherwise. There are two types of convergence used with the Mikusiński operators. In the usual type of convergence, which we will call (I), a sequence of $f_{n} \in \mathfrak{L}$ converges to zero if and only if there exists a $\phi \neq 0$ such that $\phi f_{n} \rightarrow 0$ in the $\mathscr{L}$ topology. In the second type of convergence, which we will call (II), a sequence $f_{n}$ in $\mathscr{L}$ converges to zero if and only if there exists a sequence of $\phi_{n}$ which converge to $\phi \neq 0$ in $\mathscr{L}$ and $\phi_{n} f_{n}$ converges to zero in $\mathscr{L}$. In Urbanik [5] it is shown that the sequence $\left\{e^{n t}\right\}$ is convergent to zero with type (II) convergence. We will now show that there are increasing sequences of positive functions which converge to zero with type (I) convergence, and in fact, there is such a sequence convergent to any integrable function.

THEOREM. The following four conditions on a real $\phi$ are equivalent:

(i) $\phi$ can be made positive by convolution;

(ii) every sequence $p_{n} \geqq 0$ in $\&$ such that $\phi p_{n}$ is convergent in $\mathscr{L}$ is bounded in \&;

(iii) the closure in $\mathscr{L}, \mathrm{Cl}(\phi \mathcal{P})$, of the set $\phi \mathcal{P}=\{\phi p \mid p \in \mathcal{L}, p \geqq 0\}$ is the set $\phi \mathfrak{M}=\{\phi \mu \mid \mu$ is non-negative Baire measure $\}$;

(iv) the set $\phi \mathcal{P}$ is not dense in $\mathcal{L}$.

We shall prove the theorem by means of several lemmas. In $\$ 4$ an example is given of a function which cannot be made positive by convolution.

LemMa 1. Let $f_{n}$ be a bounded sequence in $\&$ and suppose $\phi f_{n}$ is convergent in $\&$. Then $f_{n}$ is weak star convergent to a locally finite Baire measure $\mu$ and for all $g$ in $\&$ the sequence $g f_{n}$ converges in $\&$ to $g \mu$.

Proof. The sequence $f_{n}$ has a weak star limit point $\mu$ and the fact that $\phi f_{n}$ is convergent in $\mathscr{L}$ shows that $f_{n}$ has only one limit point. The $g f_{n}$ converge to $g \mu$ in $\mathscr{L}$ since the pointwise limit and the $\mathscr{L}$ limit must be the same.

Lemma 2. Let $p_{n}, n \geqq 0$, be a sequence of non-negative functions in $\mathfrak{L}$. If $\phi$ can be made positive by convolution, and if $\phi p_{n}$ is convergent in $\mathfrak{L}$, then $p_{n}$ is weak star convergent to a non-negative Baire measure $\mu$ and for each $g$ in $\mathscr{L}, g p_{n}$ converges in $\mathfrak{L}$ to $g \mu$.

Proof. Since the weak star limit of non-negative functions is a non-negative measure, in view of Theorem 1 it is only necessary to show that the $p_{n}$ are bounded. An easy argument shows that if $\phi$ can be made positive by convolution, the $p_{n}$ must be bounded in $\mathcal{L}$.

LEMma 3. If there exists a sequence $p_{n} \geqq 0$ which fails to be bounded in 
$\mathcal{L}$, but $\phi p_{n}$ is convergent in $\mathscr{L}$, then there exists a sequence $q_{n}$ such that for every $t>0$ the numbers $\int_{0}^{t} q_{n} \rightarrow \infty$ as $n \rightarrow \infty$ but $\phi q_{n} \rightarrow 0$ in $\mathfrak{L}$.

Proof. If the hypothesis of the theorem holds there must be a sequence $p_{n}, n \geqq 0$, of non-negative functions in $\mathscr{L}$ such that $\phi p_{n}$ is convergent to zero in $\mathscr{L}$ as $n \rightarrow \infty$, but such that $p_{n}$ is not a bounded sequence in $\mathscr{L}$. Let

$$
T=\operatorname{Sup}\left\{t \mid \text { there exists } A>0 \text { such that } \int_{0}^{t} p_{n}<A \text { for all } n \geq 0\right\} .
$$

If $T=0$ we can take the desired sequence $q_{n}, n \geqq 0$, to be a subsequence of the sequence $p_{n}, n \geqq 0$. If $T>0$, for each $\epsilon$ in $(0, T)$ there is a real number $A_{\epsilon}$ such that $\int_{0}^{t-\epsilon} p_{n}<A_{\epsilon}$ for all $n \geqq 0$. Define $l_{n, \epsilon}$ and $r_{n, \epsilon}$ by

$$
l_{n, \epsilon}(t)= \begin{cases}p_{n}(t), & t \in[0, T-\epsilon], \\ 0, & t>T-\epsilon\end{cases}
$$

and

$$
r_{n, \epsilon}(t)= \begin{cases}0, & t \in[0, T-\epsilon], \\ p_{n}(t), & t>T-\epsilon .\end{cases}
$$

Since $\left(\phi p_{n}\right)(t)=\left(\phi l_{n, \epsilon}\right)(t)$ on $[0, T-\epsilon]$ it follows that the sequence $l_{n, \epsilon}, n \geqq 0$, converges as Baire measures on $[0, T-\epsilon]$ to zero in the weak star topology. Since $l_{n, \epsilon}(t)=0$ for $t>T-\epsilon$ the sequence converges in the weak star topology to zero on the entire interval $t \geqq 0$. Thus the non-negative, nondecreasing functions $h l_{n, \epsilon}$ converge to zero for each $t \geqq 0$ as $n \rightarrow \infty$. Lebesgue's bounded convergence theorem, $\phi h l_{n, \epsilon}$ converges to zero in $\mathscr{L}$ as $n \rightarrow \infty$ and thus $\phi h r_{n, \epsilon}=\phi h p_{n}$ - $\phi h l_{n, \epsilon}$ converges to zero in $\mathfrak{L}$ as $n \rightarrow \infty$ for each $\epsilon$. Since $r_{n, \epsilon}(t)=0$ on $[0, T-\epsilon], n \geqq 0$, we can define the functions $q_{n, \epsilon}$ by $h r_{n, \epsilon}=e^{-(T-\epsilon) s} q_{n, \epsilon}$, $n>0, \epsilon$ in $(0, T)$. Then $\phi q_{n, \epsilon}$ converges to zero in $L$ as $n \rightarrow \infty$ for each $\epsilon$ but $\int_{0}^{2 \epsilon} q_{n, \epsilon}=A_{\epsilon}(n)$ is an unbounded sequence for each $\epsilon$ in $(0, T)$. By taking a countable number of such sequences, say with $\epsilon=1 / k$, $k=1,2, \cdots$, and choosing an appropriate diagonal sequence $q_{n}$ we get a sequence with the properties stated in the lemma.

We are now ready to begin the proof of our theorem.

Proof of Theorem. Lemma 2 shows that (i) $\Rightarrow$ (ii) and that (ii) $\Rightarrow$ implies $\mathrm{Cl}(\phi \odot) \subset \phi \Re$. A routine argument shows that each element of $\phi \mathscr{T}$ is the limit in $\mathscr{L}$ of a sequence from $\phi \mathcal{P}$ and thus $\mathrm{Cl}(\phi \mathcal{P})=\phi \mathscr{M}$, which is (iii). Clearly (iii) implies (iv). Thus (i) $\Rightarrow$ (ii) $\Rightarrow$ (iii) $\Rightarrow$ (iv).

We will show that (iii) and (iv) each imply (ii). Suppose that (ii) fails to hold. Let $q_{n}$ be as in Lemma 3 . The functions $e_{n}$ defined by $e_{n}(t)=\exp \left[-\left(h q_{n}\right)(t)\right]$ converge to zero in $\mathcal{L}$ by Lebesgue's bounded 
convergence theorem, and thus $\phi e_{n} \rightarrow 0$ in $\mathscr{L}$ as $n \rightarrow \infty$. For $t \geqq 0$, $e^{-t}-(1-t) \geqq 0$, so that the functions $r_{n}$, defined by $e_{n}=h-h q_{n}+r_{n}$, are non-negative and $\phi r_{n}=\phi e_{n}-\phi h+\phi q_{n}$ converges in $\mathscr{L}$ to $-\phi h$ as $n \rightarrow \infty$. Thus (iii) fails to hold.

Moreover, since $\phi r_{n} h^{k} \rightarrow-\phi h^{k+1}$ as $n \rightarrow \infty$ for each $k=0,1,2, \ldots$ (and since $\phi h^{k} \in \phi \odot$ for each $\left.k \geqq 1\right) \phi P \in \mathrm{Cl}(\phi \odot)$ for every real polynomial $P$. Since the polynomials are dense in $\mathscr{L}$ we have $\phi \mathfrak{L} \subset \mathrm{Cl}(\phi \odot)$ and since $\phi \mathfrak{L}$ is dense in $\mathscr{L}$ we have $\mathscr{L} \subset \mathrm{Cl}(\phi \odot)$ which proves that (iv) $\Rightarrow$ (ii).

Thus, (ii), (iii) and (iv) are equivalent, and it remains only to show that one of these implies (i). We shall show that (iii) implies (i) by means of the following lemma which follows immediately from the Hahn-Banach Theorem [6].

Lemma 4. Let \& be a Hausdorff locally convex topological vector space over the real numbers. If $C$ is a closed convex cone in $\&$ which contains no nontrivial subspace of $\mathcal{E}$ there is a nontrivial linear functional $F$ on $\mathcal{E}$ which is non-negative on $C$.

To show that (iii) implies (i) we note that the closed convex cone $\phi \mathfrak{M}$ of (iii) contains no element $-\phi \mu, \mu \in \mathfrak{M}$, and according to Lemma 4 there is a nontrivial real linear functional $F$ on the vector space of real valued locally integrable functions (over the real field) which is non-negative on $\phi M$; there is a corresponding real function $b$ which vanishes for $t$ greater than some $T$ and is essentially bounded on $[0, T]$ which determines the linear functional. In particular,

$$
\begin{aligned}
F\left(\phi e^{-\lambda s}\right) & =\int_{0}^{\infty}\left(\phi e^{-\lambda s}\right)(t) b(t) d t, & & \lambda \geqq 0, \\
& =\int_{\lambda}^{T} \phi(t-\lambda) b(t) d t, & & 0 \leqq \lambda \leqq T, \\
& =\int_{0}^{T-\lambda} \phi(T-\lambda-u) b(T-u) d u \geqq 0, & & 0 \leqq \lambda \leqq T .
\end{aligned}
$$

Thus the function $b_{1}$ defined by $b_{1}(t)=b(T-t)$ on $[0, T]$ and $b_{1}(t)=0$ for $t>T$ is such that the function $\phi b_{1}$ is non-negative on $[0, T]$. Let $\tau=\operatorname{Sup}\left\{t \mid b_{1}(u)=0\right.$ a.e. on $\left.[0, T]\right\}$ then $\tau<T$ since $F \neq 0$, and the function $b_{2}$ defined by $b_{2} e^{-\tau e}=b_{1}$ vanishes on no neighborhood of the origin, while the continuous function $\phi b_{2}$ is non-negative on $[0, T-\tau]$ so that $\phi h b_{2}$ is nondecreasing on $[0, T-\tau]$ and strictly positive on $(0, T-\tau)$. A familiar type of argument shows that there are constants $A_{n}$ increasing sufficiently rapidly so that 


$$
k=h b_{2} \sum_{n=0}^{\infty} A_{n} e^{-n(T-\tau) s}
$$

has the property that $(\phi k)(t)>0$ for $t>0$.

In the case of a complex $\phi$ the Theorem needs to change only in that (i) reads "there exists a (possibly complex valued) nonzero function $k$ such that $\operatorname{Re} k \phi(t) \geqq 0$," and (iv) reads " $\phi \mathscr{L}$ is properly contained in $\mathrm{Cl}(\phi \odot)$." Here $\mathcal{L}$ is still the real vector space of real valued functions.

4. An example. The condition that $\phi$ can be made positive by convolution is not a vacuous condition; there exist real functions which cannot be made positive by convolution.

ExAmple. The function $f(t)=t^{-1 / 2} \sin (1 / t)$ has a Laplace transform $\hat{f}(x)=\int_{0}^{\infty} f(t) e^{-x t} d t$ which is given by $[7$, p. 254, line 9$]$

$$
\hat{f}(x)=\sqrt{ } \pi \frac{e^{-\sqrt{ } 2 x} \sin \sqrt{ } 2 x}{\sqrt{ } x}, \quad x>0,
$$

and this has zeros arbitrarily far out on the real axis. Thus for any function $k_{1}$ which has a Laplace transform, $\hat{f}(x) \hat{k}_{1}(x)$ has zeros arbitrarily far out on the real axis. On the other hand, if $\phi$ is any function which can be made positive by convolution with $k$, the function $k_{0}$ obtained from $k$ by taking $k_{0}(t)=k(t)$ for $t \in[0,1], k_{0}(t)=0$ for $t>1$, is such that $r=\phi k_{0}$ is strictly positive on $(0,1) . k$ can be taken to be continuous so that $k_{0}$ is bounded, and if $\phi$ has a Laplace transform, then $\hat{r}(x)=\hat{\phi}(x) \hat{k}_{0}(x)$ is strictly positive for sufficiently large real $x$. Clearly there is no $k_{0}$ such that $\hat{f}(x) \hat{k}_{0}(x)$ is strictly positive for all $x$ in a neighborhood of infinity and thus $f$ cannot be made positive by convolution.

\section{RefERENCES}

1. E. C. Titchmarsh, On the zeros of certain integral functions, Proc. London Math. Soc. (2) 25 (1926), 283-302.

2. C. Foias, Approximation des operateurs de J. Mikusinski par des fonctions contives, Studia Math. 21 (1961), 73-74.

3. G. K. Kalisch, A functional analysis proof of Titchmarsh's theorem on convolution, J. Math. Anal. Appl. 5 (1962), 176-183.

4. T. K. Boehme, On approximate solutions to the convolution equation on the halfline, Bull. Amer. Math. Soc. 6 (1963), 847-849.

5. K. Urbanik, Sur la structure non topologique du corps des operateurs, Studia Math. 14 (1954), 243-246.

6. N. Bourbaki, Espaces vectoriels topologiques, Actualités Sci. Ind., No. 1189, Hermann, Paris, 1953.

7. A. Erdélyi et al., Tables of integral transforms, Vol. I, McGraw-Hill, New York, 1954.

University of California, Santa Barbara 